\title{
The complex of San Lorenzo del Chagres in Panama: historical development and survey project for the documentation of the Caribbean fortress
}

\author{
Sandro Parrinello ${ }^{a}$, Francesca Picchio ${ }^{b}$
}

âDipartimento di Ingegneria Civile e Architettura, Università degli Studi di Pavia, sandro.parrinello@unipv.it,

bDipartimento di Architettura, Università degli Studi di Firenze, francesca.picchio@unifi.it

\begin{abstract}
The aim of this research project was the documentation of the San Lorenzo del Chagres fort using integrated survey instruments: thanks to a total station and a camera has been possible to take pictures aiming at virtually modelling the whole building and its architectural elements present in the site. The methodology of structure from motion allows to obtain a reliable geometric model of each part, starting from a sequence of pictures, in witch is integrated the quality of materic aspect given from the texture.

Therefore, San Lorenzo del Chagres fort is a case study for new frontier of the representation: starting from a 3D model, acquired through a fast survey, it is possible to obtain each information about the state of preservation of the building with a digital database of the walls, according to the policy of conservation, monitoring and enhancement of that UNESCO cultural heritage.
\end{abstract}

Keywords: Antonelli, Structure from motion, integrated survey, documentation, 3D models.

\section{Introduzione}

Il contributo qui presentato fa parte di un progetto di ricerca più ampio, finalizzato a documentare le opere monumentali degli Antonelli, una famiglia di ingegneri militari e architetti che, al servizio della Corona Spagnola, determinarono le strategie di insediamento e lo sviluppo di sistemi difensivi del Nuovo Mondo nel Mar dei Caraibi ${ }^{1}$. A questo progetto appartiene la pianificazione di un complesso di fortezze, di cui il San Lorenzo di Panama risulta essere una delle principali roccaforti difensive, a guardia delle rotte commerciali per proteggere $\mathrm{i}$ principali porti spagnoli.

Dove il fiume Chagres sfocia nell'Oceano Atlantico fu realizzata una fortezza di notevoli dimensioni, a difesa di quello che rappresentava, per i conquistatori spagnoli, la via più breve per raggiungere la costa pacifica e le terre, ricche d'oro, nelle americhe del sud. Nel corso dei secoli il forte fu vittima di numerosi attacchi da parte dei pirati che ne distrussero gran parte della della struttura, che comprendeva oltre al sistema difensivo costituito dalla fortezza e da altre batterie, la cittadina di San Lorenzo, il cui centro si trovava alle spalle della fortezza e che si estendeva fino alle capanne dei pescatori ubicate più in basso, al lato della baia alla foce del fiume gli edifici monumentali della dogana, ubicata lungo la costa sull'altra sponda del fiume. L'esperienza di rilevamento svolta sul campo, in collaborazione tra le Università italiane e le soprintendenze locali ${ }^{2}$, descrive le fasi di acquisizione e di postproduzione dati riguardanti il complesso monumentale del San Lorenzo del Chagres. Il progetto, finalizzato alla documentazione di un sistema che da anni anima un crescente interesse da parte degli istituti di ricerca panamensi, ha avuto l'intento di 
sviluppare un programma di documentazione dettagliato e polivalente.

Da una parte è stata prevista la realizzazione di elaborati capaci di descrivere accuratamente lo stato di fatto dell'opera architettonica, elaborati necessari alla programmazione delle operazioni di salvaguardia, restauro o mautenzione; dall'altra attraverso un sistema multimediale che, virtualizzando il manufatto, rendesse disponibile l'interazione tra edificio ed utenza, non necessariamente specializzata nel settore.

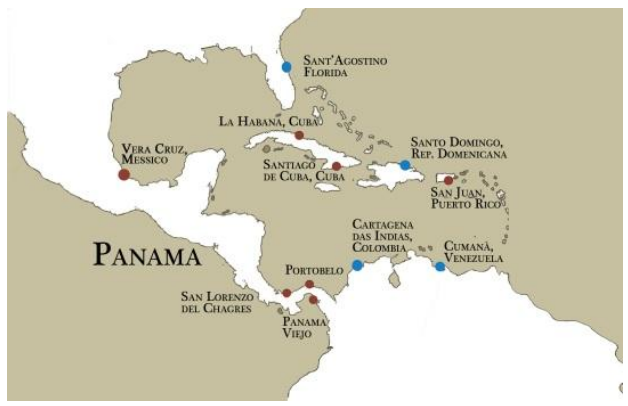

Fig. 1- Mappa del Centro America nella quale sono riportate in rosso le fortezze rilevate ed in blu quelle ancora da rilevare

\subsection{Dalle origini all'attuale immagine del Forte San Lorenzo}

Durante l'epoca della conquista spagnola le acque navigabili del fiume Chagres facevano parte del Camino de Cruces, che attraversava l'istmo unendo la costa caraibica e quella pacifica. Nel 1595, per difendere la foce del rio dai pirati, Filippo II di Spagna fece costruire, su di una ripida scogliera, il castello di San Lorenzo. Come è possibile osservare dalle cartografie storiche (Fig. 2) al lato del forte, che presidiava la foce del fiume, sorgeva anche un'importante città (anch'essa in parte fortificata) e un villaggio che si estendeva lungo le sponde. Al termine del canale di navigazione fluviale era presente una dogana, sul versante opposto al forte.

Nonostante l'imponenza e il luogo strategico sul quale sorgeva, nel 1596 il pirata Frencis Drake conquistò il castello, precedendo di quasi un secolo l'attacco di Henry Morgan, che poi si diresse alla conquista Città di Panama.
Il forte subì un ulteriore devastante attacco nel 1680 per mano dell'ammiraglio britannico
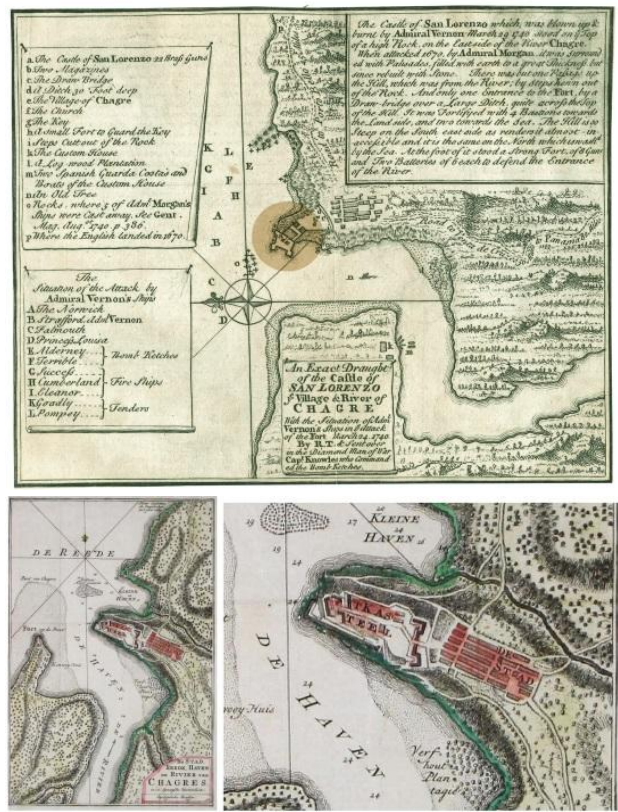

Fig. 2- Planimetria del Forte con evidenziato il sistema insediativo territoriale, in particolare il tracciato compatto con il quale viene disegnata la città di San Lorenzo.

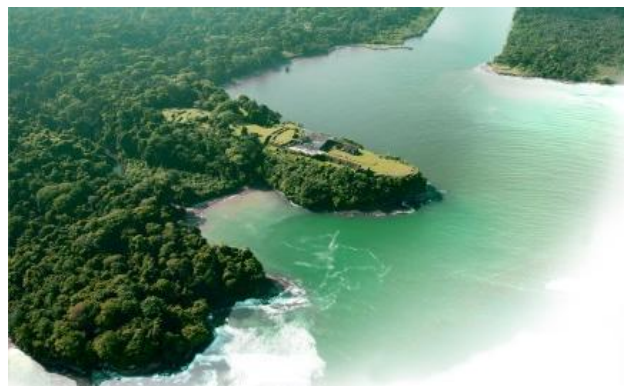

Fig. 3- Veduta aerea del forte di San Lorenzo sulla foce del Chagres. (J. Kingston Photo)

Il sistema collinare limitrofo al forte era, come dimostrano le immagini storiche, completamente privo di vegetazione ad alto fusto per non occludere le visuali di avvistamento sia sul mare che verso terra. Ad eccezione dela piazza d'arme e della zona retrostante l'ingresso alla fortezza, in passato occupate dalle case degli schiavi, le altre zone dell'insediamento spagnolo sono interamente scomparse sotto la foresta. 

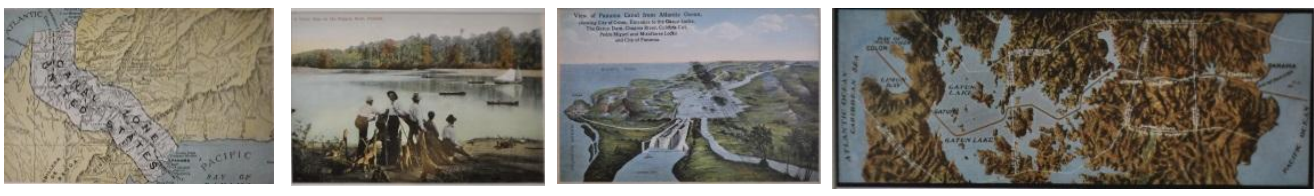

Fig. 4- Immagini storiche, risalenti all'inizio del secolo, relative alla costruzione del canale.

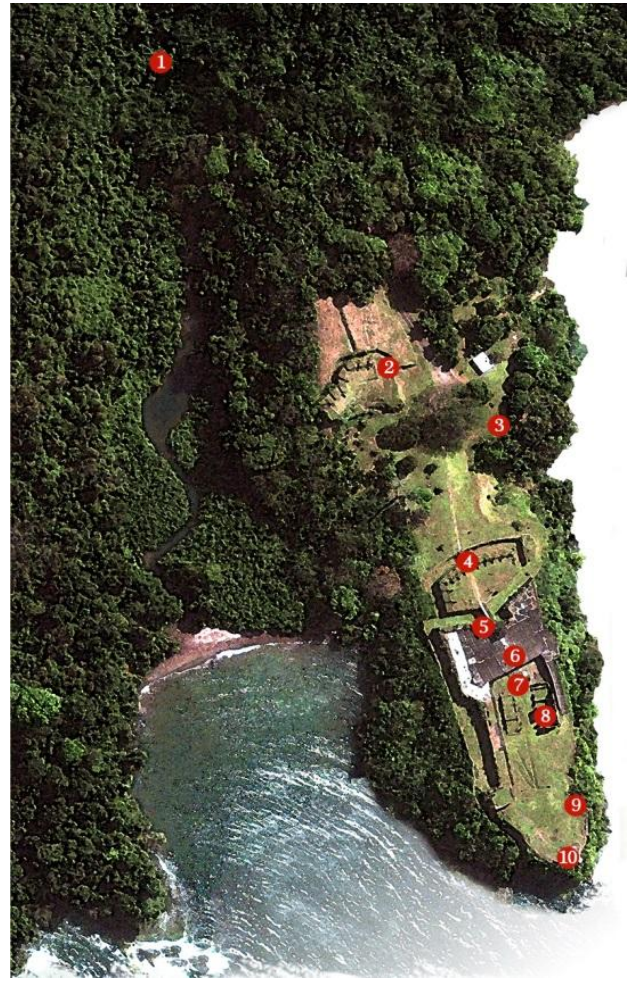

Fig. 5- Fotografia aerea del complesso del San Lorenzo.

Legenda

1.Rovine della città di San lorenzo

2.Batteria di Terra

3.Resti delle abitazioni e area dei reperti in ceramica

4.Ponte di accesso al primo fossato

5.Ponte di accesso al forte e ingresso

6.Ambienti coperti per le truppe

7.Cisterna

8.Casa del capitano

9.Resti di batterie

10.Batteria di testa

Zdward Vernon e, dal 1761, anno della sua ultima ricostruzione, il castello fu scarsamente alterato, a seguito del cambio delle rotte commerciali che ne fecero perdere interesse all'interno della più ampia strategia commerciale delle americhe. Nel 1821, con l'indipendenza di Panama, il forte fu abbandonato e poi utilizzato dapprima come prigione dall'esercito Colombiano, poi come ufficio postale per la corrispondenza proveniente dall'inghilterra. $\mathrm{La}$ costruzione del Canale e la difesa della Canal Zone implicò un diverso sfruttamento della giungla circostante da parte dell'esercito americano, che fece costruire un grande forte militare, Fort Sherman, seguito da altri forti più piccoli che, nel 1999 col trattato di Torrijos Carter, tornarono sotto la giurisdizione panamense. Dal 1980 il Castello viene dichiarato Patrimonio dell'Umanità dall'UNESCO ${ }^{3}$.

\section{Modalità di acquisizione dei dati}

Al fine di verificare la presenza di ruderi o resti di eventuali insediamenti lungo le sponde e nell'entroterra della foresta che circonda il Rio Chagres, sono state effettuate escursioni e ricognizioni via mare e via terra. In particolare sono stati verificati quei siti che, nelle cartografie storiche, erano descritti con nuclei insediativi o sistemi difensivi.

Le ricognizioni effettuate attorno all'area visitabile della fortezza, hanno consentito di comprendere con maggior consapevolezza la dimensione territoriale del sito e le conformazioni di tale territorio. Pendii scoscesi ricoperti da una fitta vegetazione di giungla hanno in qualche misura vincolato la scelta della strumentazione più efficace per acquisire dati di natura metrica in grado di definire strumenti che permettessero di restituire la forma e l'immagine del sistema architettonico. Nella zona limitrofa alla fortezza, sviluppata su una piattaforma pianeggiante, il critico stato di conservazione rende il manufatto edilizio difficilmente accessibile per gran parte dei suoi ambienti. 


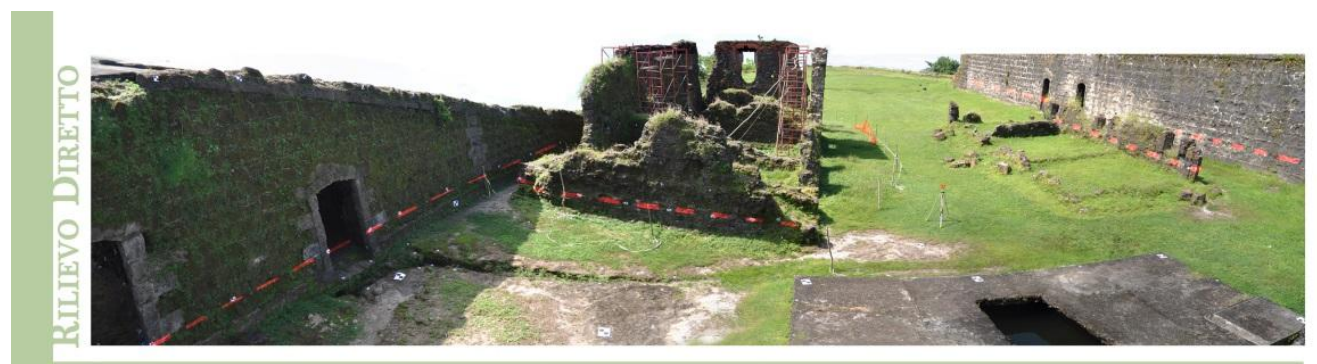

Dalle stazioni topografiche sono stati misurati i target disposti sulla forteza ed i punti notevoli degli elementi architetonici.
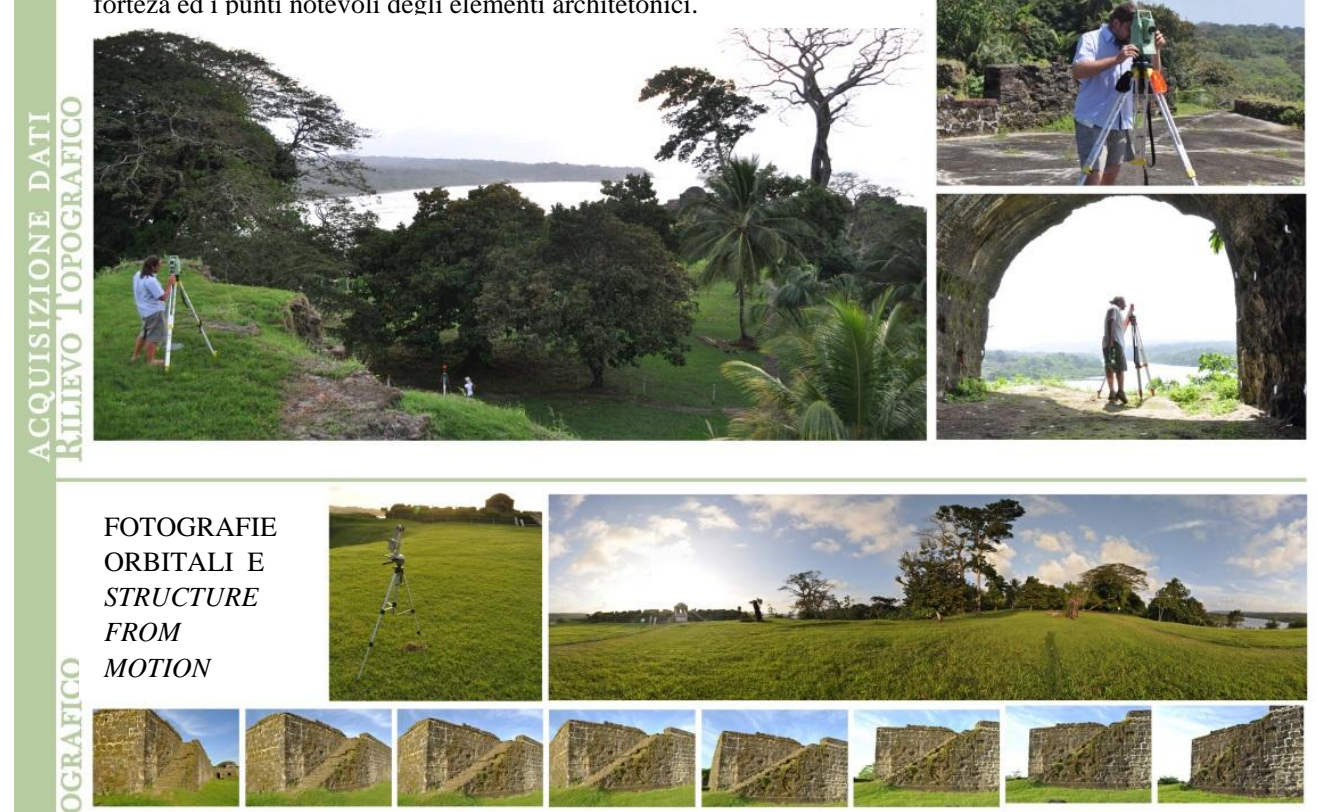

Con delle machine fotografiche, attraverso procedimenti structure from motion, sono stati ricostruiti gli ambienti virtuali utilizzando come strumento di verifica il rilievo topografico eseguito per ciascun ambiente, alineando le nuvole di punti e i modelli 3D ai target della nuvola di punti topografica.

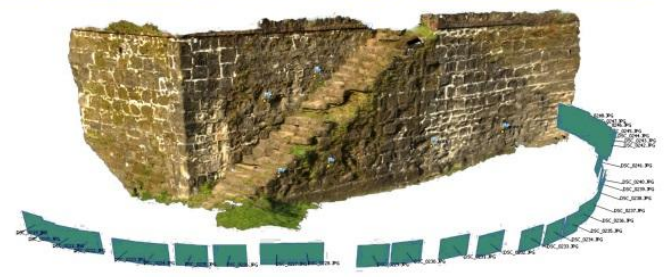

Pertanto la complessità del sito, sia per quanto riguarda l'accessibilità degli ambienti, sia relative alla complessità specifica delle singole murature (per la maggior parte presentanti discontinuità superficiali per l'avanzata fase di deterioramento o il parziale crollo di alcune porzioni), ha vincolato la scelta della strumentazione da utilizzare. La necessità di ottenere un prodotto metricamente e

qualitativamente affidabile nel breve tempo a disposizione per eseguire la campagna di rilievo, ha portato a scegiere una procedura di acquisizione dati che, tramite strumento fotografico, fosse in grado di elaborare modelli tridimensionali altamente affidabili, capaci di descrivere e riportare le specificità di ogni ambiente $^{4}$. A tale metodologia di acquisizione dati è stata affiancata la pianificazione di un 
accurato rilievo topografico. Le misurazioni topografiche, utilizzate sia per rilevare alcune delle misure fondamentali che costituiscono l'oggetto, sia per elaborare una nuvola di punti molto rada dell'intero complesso, sono state impiegate per referenziare rispetto ad un'unica terna cartesiana i singoli modelli provenienti dalle sequenze fotografiche.

In un contesto così configurato l'immagine fotografica assume un ruolo decisivo: essa diventa un descrittore esaustivo dello spazio, fondamentale per definire le qualità ambientali di un luogo integrando le informazioni e rendendo univoca la lettura e la comprensione delle relazioni presenti su un territorio anche ad utenti di culture che utilizzando linguaggi e forme di comunicazioni differenti. Uno spazio tridimensionale ottenuto da tale metodologia garantisce molteplici finalità. Nell'ambito dell'analisi finalizzata alla comprensione delle relazioni esistenti tra gli elementi della scena, sia in merito alla documentazione ed alla conservazone del patrimonio per fornire strumenti valutativi utili nel campo del restauro.

\subsection{Scomposizione e ricomposizione del sistema}

Utilizzare il prodotto della campagna fotografica, ovvero i modelli tridimensionali da essa generati, come elemento sul quale strutturare gli elaborati di rilievo ha previsto una precisa e sistematica organizzazione della fase di acquisizione dati. Ciascuna area del complesso è stata suddivisa in porzioni al fine di determinare una struttura semantica utile alla costruzione di un archivio che risultasse utile alla strutturazione dei dati in relazione alle attività di rilievo programmate. I criteri di scomposizione del complesso in elementi di più facile gestione, sono dipendenti dalle caratteristiche morfologiche dell'intero sistema architettonico.

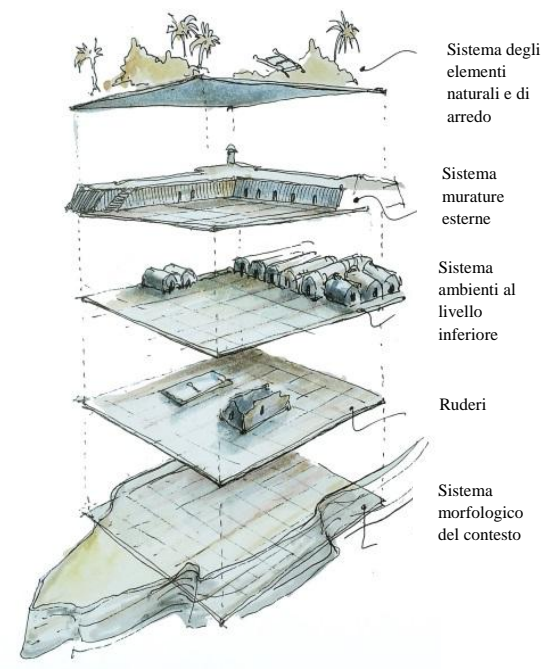

Fig. 6- Struttura del sistema di scomposizione per livelli del complesso.

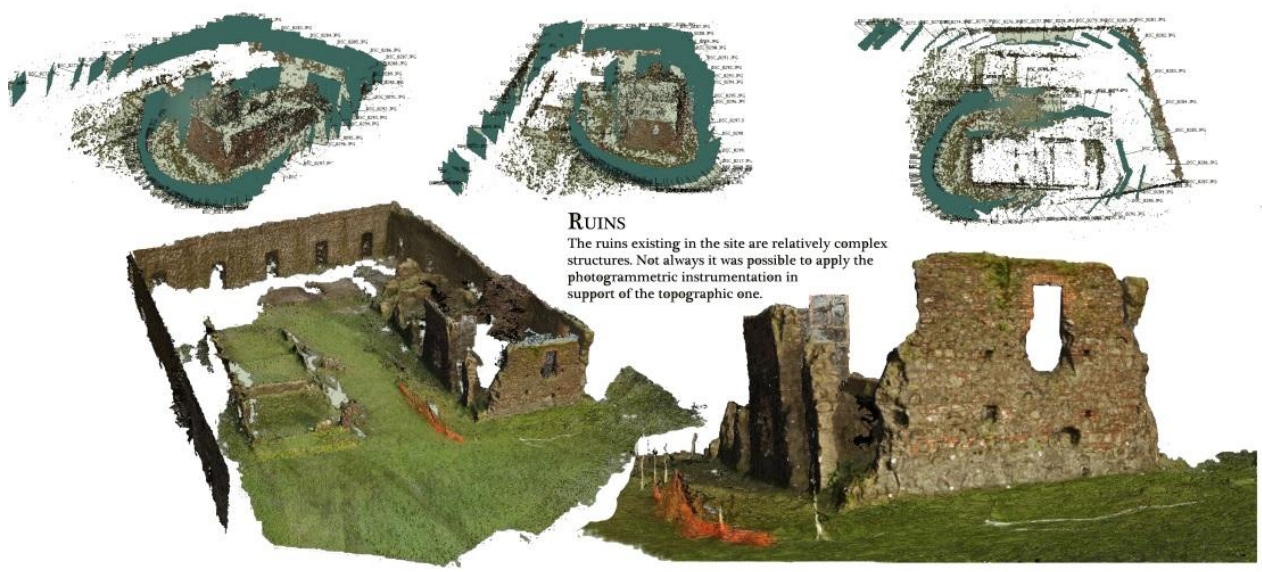

Fig. 7- Shape from motion dei ruderi della Casa del Capitano sul piazzale posteriore del complesso. 


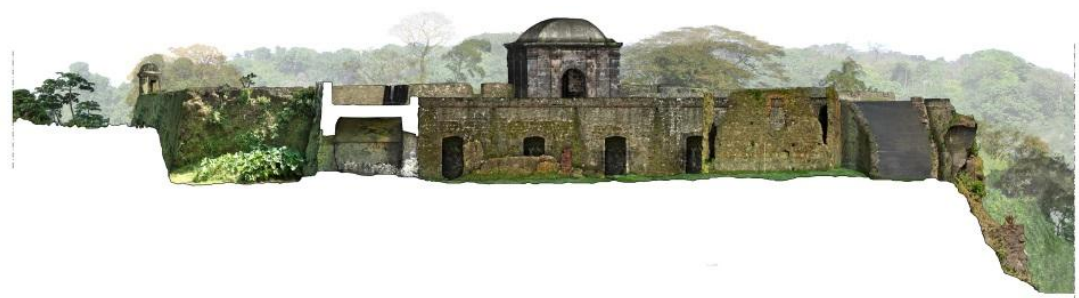

Fig. 8- Sezioni ambientali del forte e del suo contesto. L'elaborazione delle sezioni è stata impostata sulla base del modello fotografico tridimensionale, ricomponendo le singole porzioni in cui il modello era stato scomposto in fase di acquisizione.

Una prima macro divisione ha scomposto l'oggetto su due livelli, uno inferiore e uno superiore. Il piano inferiore, che presenta numerosi ambienti indipendenti e concatenati, ha previsto un' ulteriore discretizzazione della struttura classificando ogni rudere, stanza o paramento murario con schemi grafici e codici identificativi. Le caratteristiche di ogni ambiente sono state dapprima esplicate attraverso il disegno: il disegno, oltre a evidenziare le qualità spaziali di ciascun ambiente, ha consentito di analizzare le criticità della struttura e fornire da base per la scomposizione del sistema generale in unità murarie. A seguito di questo processo è stato possibile catalogare ciascun elemento con codici identificativi che permettessero una programmazione ordinata della campagna fotografica. Parallelamente alla realizzazione del rilievo topografico, la campagna fotografica sviluppata per coprire interamente l'oggetto architettonico ha costretto a pensare a sistemi funzionali di archiviazione dati che hanno visto l'organizzazione di un sistema ad albero nel quale ogni paramento murario, dotato di codice identificativo progressivo, fosse catalogato e provvisto del numero di fotografie necessarie per il suo sviluppo tridimensionale.

Questa operazione, necessaria in fase di acquisizione per scomporre il problema in complessità più facilmente gestibili, è servita anche a semplificare la successiva fase di elaborazione dei dati acquisiti. Nella pratica tradizionale da elaborati di tipo bidimensionale, (planimetrie e sezioni) vengono costruiti modelli tridimensionali nei quali le informazioni, per questioni logistiche legate alla gestione del dato, vengono decisamente ridotte e semplificate all'essenziale dell'ingombro volumetrico, a scapito, però, di tutti quei dati relativi alla composizione superficiale dei singoli paramenti.

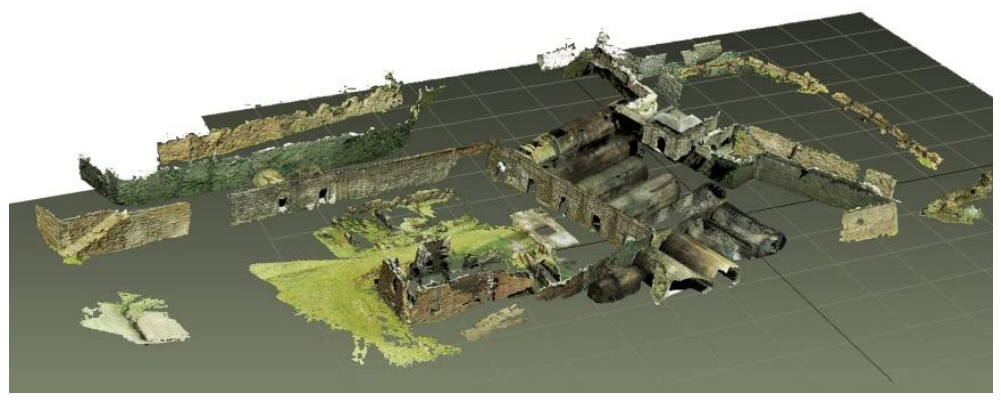

Fig. 9- Step della ricomposizione del livello inferiore degli ambienti nel modello generale

Per il rilievo del forte del San Lorenzo è stato sviluppato un processo inverso. Il modello realizzato è composto da due sistemi perché duplice ne è la finalità. Il primo sistema riguarda il modello completo dell'intero forte, dove dal contesto tridimensionale reality based, altamente descrittivo e non discretizzato, avviene una sostanziale semplificazione dei dati acquisiti. La finalità di tale modello è la fruizione via web, la virtualizzazione del sistema che, pertanto, 
risponderà a requsiti di leggerezza del file, facilità di gestione e rispondenza suggestivaall'immagine reale dell'ambiente. Il modello viene notevolmente semplificato nei dati, elaborato al fine di rimuovere le imperfezioni della maglia mesh della struttura e esportato in software capaci di simulare, attraverso librerie naturali, il contesto virtuale nel quale il modello si inserisce. L'altra finalità è, invece, quella di realizzare un sistema di modelli in cui la semplificazione delle maglie triangolari non è avvenuta, al fine di matenere inalterate le caratteristiche di struttura e di qualità del dato emerso dalla campagna fotografica la metodologia condotta è stata elaborata al fine di dotare ogni modello delle informazioni necessarie ad una corretta lettura dello stato di conservazione di ogni unità muraria.

\section{Conclusioni}

L'esperienza qui riportata fa parte di un progetto che, ormai da diversi anni, ha la finalità di sperimentare su molteplici siti architettonici l'applicazione della metodologia structure from motion e la gestione dei dati acquisiti su complessi di notevole vastità ${ }^{5}$. A questo proposito sarà utile notare come, qui come in molti altri sistemi architettonici complessi, risulti fondamentale strutturare a priori un sistema di discretizzazione, catalogazione e archiviazione degli elementi costituenti il manufatto. Queste operazioni fanno tutte capo ad un sistema necessario di decodificazione dello spazio, riconducibili alle più tradizionali e consolidate forme del disegno. E' attraverso il disegno che viene strutturata una corretta scomposizione dello spazio, dapprima compreso e, solo successivamente, acquisito. Le modalità in cui il soggetto percipiente elabora le informazioni provenienti dall'oggetto percepito fanno parte di quell'ambito della comunicazione in cui è proprio il disegno a risultare, ancora più efficacemente dell'immagine fotografica, il mezzo più chiaro di decodificazione dello spazio. Una ricerca che riesca a comprendere la natura di come le informazioni vengono disposte ed elaborate dall'osservatore è, con molte probabilità, un filone per organizzare una campagna fotografica più consapevole ed efficace.Solo in questo modo il modello potrà acquisire la valenza di organizzatore dello spazio, sistema di comunicazione efficace e non discutibile, superando non solo la rappresentazione classica 2D, ma configurandosi come valida supporto al disegno stesso ${ }^{6}$.

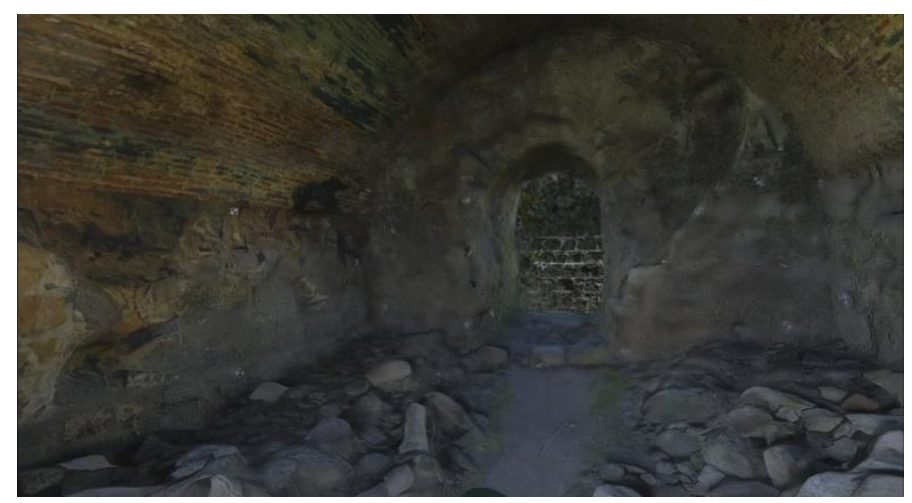

Fig. 10- Vista da un software real time (Lumion) di uno degli ambienti del modello virtuale decimato ed ottimizzato nella struttura delle maglie mesh.

\section{Notes}

(1) Il progetto di ricerca per la documentazione delle architetture fortificate realizzate su progetti della famiglia Antonelli, coordinato da Sandro
Parrinello, prende avvio nel 2005 con la campagna di rilievo del forte San Pedro de la Roca a Santiago de Cuba. Negli otto anni successivi sono state documentate le fortezze dell'isola cubana in accordo con l'Oficina del 
Conservador di Santiago de Cuba e l'Oficina dell'Historeador della città dell'Havana, le fortezze della città di San Juan di Porto Rico (USA), Il forte San Juan de Ulua a Veracruz (Messico), Il forte di Bernia in Spagna, la cittadella di Peniscola (Spagna) ed i siti che si trovano a Panama: Portobello, San Lorenzo, del quale riportiamo alcuni risultati in questo articolo e Panama Vieja.

(2) Le ricerche per la documentazione dei siti antonelliani a Panama sono state coordinate da Sandro Parrinello in collaborazione con le istituzioni governative locali. In particolare per il Patronato de Portobelo y San Lorenzo: Nilda Quijano, Manager MIT, Manzanilla International Terminal; Yelitza Norse, ViceDirettrice del Patronato; Rodolfo Suñe, Architetto del Patronato. Per l'INAC, Instituto Nacional de Cultura: María Eugenia Herrera, direttrice generale; Almyr Alba, Architetto. Per il Parque Nacional Portobelo: Elizabeth Castro, Directtrice. Per la Fundación Bahía de Portobelo: Caridad García, Directtrice. Del Municipio di Portobelo: Carlos Chavarria Cerezo, Sindaco.

(3) Motivazioni della commissione UNESCO dell'inserimento dei complessi fortificati panamensi nella lista: A group of XVIIth and XVlllth century fortifications, the historic sites of Portobelo and San Lorenzo are outstanding examples of Spanish colonial military architecture of this period. [..] The forts, castles, barracks and batteries of Portobelo created a defensive line around the Bay and protected the harbour: the works at San Lorenzo guarded the mouth of the Chagres. conquered by Henry Morgan in 1668 and by Admiral Edward Vernon in 1739 , these fortifications were continuously rebuil because they command the access to the Panamaniarl Isthmus which has always been of the outmost importance for Europe's commerce with its colonies. International council on monltments and sites ICOMOS.

(4) Cfr. Parrinello S., Picchio F., Dalla fotografia digitale al modello 3D dell'architettura storica, in Disegnare con, a cura di Pablo RodríguezNavarro, 2013. Vol.6, n 12.

(5) Per un quadro generale delle sperimentazioni della metodologia su siti UNESCO Cfr. Bertocci S., Parrinello S., UNESCO World Heritage List, Digital Survey and Documentation of the Archaeological and Architectural sites. Edifir, Edizioni Firenze, 2014.

(6) Si devono a Sandro Parrinello i paragrafi 1, 1.1 e le conclusioni; a Francesca Picchio i paragrafi 2, 2.1 e l'abstract.

\section{References}

Benedetti B., Gaiani M., Remondino F., (2009), Modelli digitali 3D in archeologia: il caso di Pompei, (a cura di), Edizioni della Normale, Pisa.

Bertocci S., Bini M., (2012), Manuale di rilievo architettonico ed urbano, Città Studi Edizioni, De Agostini Scuola SpA, Novara.

Bertocci S., Parrinello S., (2014), UNESCO World Heritage List, Digital Survey and Documentation of the Archaeological and Architectural sites, Edifir, Edizioni, Firenze.

Merlo A., Sánchez Belenguer C., Vendrell Vidal E., Fantini F., and Aliperta A., (2013), 3D Model visualization enchancements in real-time game engines,. In Boehm J., Remondino F., Kersten T., Fuse T., Gonzalez-Aguile D. (a cura di), 3D-ARCH 2013 - 3D Virtual Reconstruction and Visualization of Complex Architectures, Trento, Italy.

Parrinello S., Picchio F., (2013), Dalla fotografia digitale al modello 3D dell'architettura storica, in Disegnare con, a cura di Pablo Rodríguez-Navarro, Vol. 6, n¹2, Bologna.

Rodriguez-Navarro P., (2012), Digital photogrammetry versus the system based on active $3 D$ sensors, in Revista EGA, número 20, Valencia. 